\title{
Formal Verification of Temporal Properties for Reduced Overhead in Grid Scientific Workflows
}

\author{
Jun-Wei Cao ${ }^{1,2}$ (曹军威), Member, CCF, ACM, Senior Member, IEEE \\ Fan Zhang ${ }^{3}$ (张 帆), Student Member, IEEE, Ke Xu (许 可), Lian-Chen Liu ${ }^{3}$ (刘连臣) \\ and Cheng $\mathrm{Wu}^{3}$ (吴 澄) \\ ${ }^{1}$ Research Institute of Information Technology, Tsinghua University, Beijing 100084, China \\ ${ }^{2}$ Tsinghua National Laboratory for Information Science and Technology, Beijing 100084, China \\ ${ }^{3}$ National CIMS Engineering and Research Center, Tsinghua University, Beijing 100084, China \\ ${ }^{4}$ Morgan Stanley, Shanghai 200002, China
}

E-mail: jcao@tsinghua.edu.cn; zhang-fan07@mails.tsinghua.edu.cn; Ke.Sh.Xu@morganstanley.com; \{liulianchen,wuc\}@tsinghua.edu.cn

Received October 29, 2010; revised September 13, 2011.

\begin{abstract}
With quick development of grid techniques and growing complexity of grid applications, it is becoming critical for reasoning temporal properties of grid workflows to probe potential pitfalls and errors, in order to ensure reliability and trustworthiness at the initial design phase. A state Pi calculus is proposed and implemented in this work, which not only enables flexible abstraction and management of historical grid system events, but also facilitates modeling and temporal verification of grid workflows. Furthermore, a relaxed region analysis (RRA) approach is proposed to decompose large scale grid workflows into sequentially composed regions with relaxation of parallel workflow branches, and corresponding verification strategies are also decomposed following modular verification principles. Performance evaluation results show that the RRA approach can dramatically reduce CPU time and memory usage of formal verification
\end{abstract}

Keywords grid computing, workflow management, formal verification, state Pi calculus

\section{Introduction}

Grid computing is becoming a mainstream technology for cross-domain management and sharing of computational resources ${ }^{[1]}$. Grid workflows ${ }^{[2]}$, a composition of various grid services according to prospective processes, have become an important paradigm for the problem solved in various scientific and industrial domains, e.g., gravitational wave data analysis ${ }^{[3]}$, biomedical simulation ${ }^{[4]}$, and banking ${ }^{[5]}$.

The quick growing complexity of grid applications and systems calls for the implementation of reliable and trustworthy grid workflows according to specific scientific criteria or business regulations, which has become an urgent research issue. In addition to existing grid enabling techniques, e.g., job scheduling, workflow enactment and resource location, various grid ensuring techniques $^{[6]}$, e.g., temporal reasoning ${ }^{[7]}$, are gaining more and more attention from grid community. These techniques are devoted to guaranteeing large scale grid workflows follow exactly requirements of domain users.

Delivering reliable web service applications using Petri-net or related techniques are fully investigated $^{[8-10]}$. Quality of services under web service configuration are discussed ${ }^{[11-12]}$. All these ensuring techniques are used to improve the compatibility and reliability of grid workflow applications. This work differentiates itself from those studies as using $\mathrm{Pi}$ calculusbased methods.

It has already been widely recognized that techniques, like formal verification based temporal reasoning ${ }^{[13-15]}$, are becoming more and more important for Web Services based systems in probing their potential errors and enhancing their reliability. How process algebras can be applied to model and reason the choreography of web services is discussed in [16]. Regarding grid system formalization, the Abstract State Machine-based formalism is applied in [17]

Regular Paper

This work is supported by the National Basic Research 973 Program of China under Grant Nos. 2011CB302805, 2011CB302505, the National High Technology Research and Development 863 Program of China under Grant No. 2011AA040501, and the National Natural Science Foundation of China under Grant No. 60803017. Fan Zhang is supported by IBM 2011-2012 Ph.D. Fellowship.

C) 2011 Springer Science + Business Media, LLC \& Science Press, China 
to distinguish grid features from traditional distributed systems. Other areas that this method could be used include software reuse and compatibility checking ${ }^{[18]}$ and scale-free web service composition or decomposition applications ${ }^{[19]}$.

$\mathrm{Pi}$ calculus was first proposed by R. Milner for its intrinsic combinability and mobility together with its natural description for open communication system ${ }^{[20]}$. This method is now accepted for its sound theoretical system and widely used in formal modeling, verification and validation. State Pi calculus, an extension of Pi calculus, is implemented in this work to further strengthen its capability to manage the life-cycle of system states. The proposed calculus not only enables the flexible abstraction and management of historical system events, but also facilitates the modeling and verification of grid service-based workflows.

While there are previous attempts in the study of grid verification techniques, the performance for verification itself is still a bottleneck for probing all potential pitfalls and errors in grid workflows. Especially for large scale and dynamically evolving scientific workflows, implementation of such formal verification processes have to be of low overhead in terms of verification time and memory demand to be applicable in real world grid environments. Performance improvement is also focused in this work. A relaxed region analysis (RRA) approach is proposed to divide-and-conquer global verification of a very large scale scientific workflow in LIGO (Laser Interferometer gravitational wave observatory) applications into local verifications on its sub grid workflow models.

Decomposition is a common technique used for handling complex systems in order to exponentially decrease system dimensions for overhead reduction. While application-specific decomposition strategies have been investigated in [21] for carrying out computational tasks in grid environments, our work is addressing a more general decomposition approach for grid workflows by studying their local and global process structures. Also we focus on how the correctness of a grid workflow, instead of the grid infrastructure itself, can be efficiently fulfilled. The RRA approach further studies how formal verification is decomposed with the decomposition of grid workflow to form a complete region analysis method. Besides, it also allows the relaxation of parallel branches in grid workflows to achieve better decomposition results and verification performance.

The approach is implemented using a Pi calculusbased formal modeling and verification environment for grid workflows with NuSMV2 ${ }^{[22]}$ as its engine. Three concrete application scenarios from gravitational wave data analysis ${ }^{[23]}$ are provided, which are currently the most classic grid-enabled scientific applications in the United States. While the complexity of a grid workflow grows exponentially when the number of its involving services and their interdependencies increases, the RRA approach can dramatically reduce overhead such as CPU time and memory usage of formal verification processes, as illustrated by the quantitative performance results included in this work.

The rest of the paper is organized as follows. In Section 2, the state Pi calculus and corresponding formalism of different structures are introduced. Section 3 provides detailed information on grid workflow modeling and the concept of standard regions. Corresponding verification decomposition is described in Section 4. The RRA approach is proposed in Section 5 and the implementation of RRA with performance evaluation results are investigated in Section 6 . Section 7 concludes the paper.

\section{State Pi Calculus and Formalism}

\subsection{State Pi Calculus}

State Pi calculus, as an extension to the original formalism framework of $\mathrm{Pi}$ Calculus, has three main features.

1) Utilize historical information to restrain and analyze process evolution.

2) Provide flexible abstraction of activities and communications in processes via administration of status proposition.

3) March general principles in WSDL to extend web services.

It is defined as follows:

$$
\begin{aligned}
P::= & \sum_{i=1}^{n} \alpha_{i}\{\text { StateExp }\} \cdot P_{i} \mid(\text { newx }) P|! P| \\
& P|Q| \phi P\left|A\left(y_{1}, \ldots, y_{n}\right)\right| 0, \\
\alpha_{i}\{\text { StateExp }\}::= & \bar{x}\langle y\rangle\{\text { StateExp }\} \mid x(\bar{y})\{\text { StateExp }\} \mid \\
& \tau\{\text { StateExp }\}, \\
\phi::= & {[x=y] \mid \text { eval }(\text { Prop }, \text { valueset }) \mid \phi \wedge \phi, } \\
\text { StateExp }::= & (\text { StateOp, S }) \mid \text { StateExp, StateExp, } \\
\text { StateOp }::= & +|-|++\mid--, \\
S::= & (\text { iden, trueset }), \\
\text { iden }::= & x \mid \text { iden.iden. }
\end{aligned}
$$

The fundamental concept of state $\mathrm{Pi}$ calculus is the name, which is used to express atomic interactive action in a system. A system in state Pi calculus evolves through the operators, including composition "|", choice "+", guard ".", match "[]", restriction "new" and replication "!". Every operator implements one kind of the relation $\Re$ : SysState $\times$ StateOp $\times S \rightarrow$ 
SysState, which is strictly defined in StateExp. The current system state SysState together with the state object (StateOp and $S$ ) evolve into a new system state SysState. All the states related implementations are new in this new method.

1) Output Action $(\bar{x}\langle\tilde{y}\rangle\{$ StateExp $\} \cdot P)$ : output $\tilde{y}$ through $\bar{x}$ with system behaviors evolving into $P$ and the system state evolved into a new state based on the predefined expression in StateExp. For example, in a communication system, ${ }^{\bar{x}}$ can be viewed as an output port and $\tilde{y}$ the output data, StateExp is the corresponding transition of the states.

2) Input Action $\left({ }^{x}(\tilde{y})\{\right.$ StateExp $\left.\} \cdot P\right):$ input ${ }^{\tilde{y}}$ through ${ }^{x}$ with system behaviors evolving into $P$ and the system state evolved into a new state.

3) Silent Action ( ${ }^{\tau}\{$ StateExp $\left.\} . P\right)$ : the system behavior evolves into $P$ with internal actions instead of interactions with the environment and the system state evolved into a new state.

4) Composition $(P \mid Q)$ : processes $P$ and $Q$ are independent, or synchronize with each other via an identical port. $Q$.

5) Choice $(P+Q)$ : unpredictable execution of $P$ or

6) March $([x=y] P)$ : if $x$ matches $y$, the system behavior evolves into $P$; otherwise no actions happen.

7) Restriction $(($ new $x) P): x$ is a new name within the process $P$.

8) Replication (!P): an infinite composition of process $P$.

\subsection{Formalism of Activities and Control Structures}

In this subsection, BPEL4WS (business process execution language for web services) is considered as an example to describe how state Pi calculus can be used for formal representation of workflow activities and control structures.

Four basic activities from BPEL4WS, Receive, Send, Invoke and Assign, can be defined as follows using state $\mathrm{Pi}$ calculus.

$$
\begin{aligned}
& \text { Receive (start, } \text { port }_{2}, \text { set, done }=_{\text {def }} \text { start }\{++ \text {, } \\
& \left.\left\{m s g \text { Port },\left\{\text { port }_{2}\right\}\right\}\right\} \cdot \operatorname{port}_{2}(v: t)\{--, \\
& \left.\left\{\text { msgPort },\left\{\text { port }_{2}\right\}\right\}\right\} . \overline{s e t}\langle v: t\rangle \cdot \overline{\text { done }}, \\
& \text { Reply }\left(\text { start, get, } \text { port }_{1}, \text { done }\right)=_{\text {def }} \operatorname{start.get}(v: t) \text {. } \\
& \overline{\text { port }_{1}}\langle v: t\rangle \cdot \overline{\text { done }} \text {, } \\
& \text { Invoke(start, get, } \text { port }_{1}, \text { port }_{2} \text {, done) } \\
& ={ }_{\text {def }} \operatorname{start.get}(v: t) \cdot \overline{p o r t_{1}}\langle v: t\rangle \cdot \operatorname{port}_{2}(s) \cdot \overline{\text { done }} \text {, } \\
& \text { Assign( start, } \text { get }_{1}, \text { set }_{2}, \text { done }_{\text {) }} \\
& ={ }_{\text {def }} \text { start.get }_{1}(v: t) \cdot \overline{\text { set }_{2}}\langle v: t\rangle \cdot \overline{\text { done }},
\end{aligned}
$$

$$
\begin{aligned}
& \text { Empty }(\text { start }, \text { done })={ }_{\text {def }} \text { start. } \overline{\text { done }} \text {, } \\
& \operatorname{Var}_{V}=_{\text {def }} \operatorname{Var}_{V_{0}}(\text { set, get }) \text {, } \\
& \operatorname{Var}_{V_{0}}(\text { set, get })=_{\text {def }} \operatorname{set}\left(x_{1}: t\right)\{++ \text {, } \\
& \left.\left\{\text { V.bSize, }\left\{x_{1}\right\}\right\}\right\} \text {. } \operatorname{Var}_{V_{1}}\left(\text { set, get, } x_{1}\right) \text {, } \\
& \operatorname{Var}_{V_{1}}\left(\text { set, get, } x_{1}\right)=_{\operatorname{def}} \operatorname{set}\left(x_{2}: t_{2}\right)\{++ \text {, } \\
& \left.\left\{\text { V.bSize, }\left\{x_{2}\right\}\right\}\right\} . \operatorname{Var}_{V_{2}}\left(\text { set, get, } x_{1}, x_{2}\right)+ \\
& \overline{\text { get }}\left\langle x_{1}: t_{1}\right\rangle\left\{--,\left\{\text { V.bSize, }\left\{x_{1}\right\}\right\}\right\} . \operatorname{Var}_{V_{0}}(\text { set, get }) \\
& \text {... } \\
& \operatorname{Var}_{V_{n}}\left(\text { set }, \text { get }, x_{1}, \ldots, x_{n}\right)=_{\text {def }} \overline{g e t}\left\langle x_{n}: t_{n}\right\rangle\{-- \text {, } \\
& \left.\left\{\text { V.bSize, }\left\{x_{n}\right\}\right\}\right\} . \operatorname{Var}_{V_{n-1}}\left(\text { set, get }, x_{1}, \ldots, x_{n-1}\right) \text {. }
\end{aligned}
$$

The formalism of six control structures in BPEL4WS, Sequence, While, Flow, Switch, Pick and Link, is described as follows.

1) Formalism of the Sequence Structure. The Sequence structure defines sequential relations among executions in a grid workflow:

$$
\begin{aligned}
& \text { Sequence }\left(f n_{d}\left(A c t_{1}\right), f n_{s}\left(A_{c t}\right)\right)==_{\text {def }} A_{c t} ; A_{1} A_{2} \\
& ={ }_{\text {def }}\left(\text { new } \operatorname{start}_{\text {Act }_{2}}\right)\left(\left\{\text { start }_{\text {Act }_{2}} \mid \text { done }_{\text {Act }_{1}}\right\} \text { Act }_{1} \mid \text { Act }_{2}\right) \text {. }
\end{aligned}
$$

2) Formalism of the While Structure. The While structure defines repeat invocation of one service or a group of services in a grid workflow under certain conditions:

$$
\begin{aligned}
& \text { While }\left(f_{\text {sd }}(\text { Act }), \text { start }_{\text {while }}, \text { done }_{\text {while }}\right) \\
& =_{\text {def }} \text { new } \text { start }_{\text {Act }} \text { done }_{\text {Act }} \\
& \text { start }_{\text {while }} .\left([ \text { eval } ( C , \{ t \} ) ] \left(\overline{\text { start }_{\text {Act }} \mid} \mid \text { Act } \mid \text { done }_{\text {Act }}\right.\right. \text {. } \\
& \left.\left.\left.\left(\overline{\text { start }_{\text {while }}} \mid \text { While }\right)\right)+[\operatorname{eval}(C,\{f\})] \overline{\text { done }_{\text {while }}}\right)\right) \text {. }
\end{aligned}
$$

3) Formalism of the Flow Structure. The Flow structure defines synchronization of parallel execution, completion among service activities and structures in a grid workflow:

$$
\begin{aligned}
& \text { Flow }\left(f n_{\text {sd }}\left(A_{c t}\right), \ldots, f n_{\text {sd }}\left(A_{1} t_{m}\right), \text { start }_{\text {Flow }}, \text { done }_{\text {Flow }}\right) \\
& =_{\text {def }}\left(\text { new start } \text { Act }_{1} \ldots \text { start }_{A c t_{m}} \text { done }_{\text {Act }_{1}} \ldots\right. \\
& \text { done }_{A c t_{m}} \text { ack ack') } \\
& \left(\text { start }_{\text {Flow }} \text {.Starter } \mid \text { Act } 1|\ldots| \text { Act } m \mid \text { Acker } \mid a c k{ }^{\prime}\right. \text {. } \\
& \overline{\text { done }_{\text {Flow }}} \text {, } \\
& \text { Starter }\left(\operatorname{start}_{A c t_{1}}, \ldots, \text { start }_{A c t_{m}}\right) \\
& =_{\text {def }} \overline{\text { start }_{A c t_{1}}}|\ldots| \overline{\text { start }_{A c t_{m}}} \\
& \text { Acker }\left(\text { done }_{A c t_{1}}, \ldots, \text { done }_{\text {Act }_{m}}, \text { ack, ack }\right) \\
& ={ }_{\text {def }} \text { done }_{A c t_{1}} \overline{a c k}|\cdots| \text { done }_{A c t_{m}} \cdot \overline{a c k} \mid \underbrace{a c k \ldots a c k}_{m} \overline{a c k^{\prime}} .
\end{aligned}
$$

4) Formalism of the Switch Structure. The Switch structure defines one conditional choice in a grid workflow: 


$$
\begin{aligned}
& \text { Switch }\left(f n_{s d}\left(A c t_{1}\right), f n_{s d}\left(A_{c t}\right), \text { start }_{\text {Switch }}, \text { done }_{\text {Switch }}\right) \\
& =_{\text {def }}\left(\text { new } \text { start }_{\text {Act }_{1}} \text { start }_{\text {Act }_{2}} \text { done }_{\text {Act }_{1}} \text { done }_{\text {Act }_{2}}\right. \text { ) } \\
& \text { start }_{\text {Switch }} \cdot\left(\left[\operatorname{eval}\left(C_{1},\{t\}\right)\right] \overline{\text { start }_{\text {Act }}}\{(++ \text {, }\right.
\end{aligned}
$$

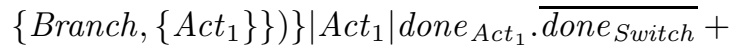

$$
\begin{aligned}
& {\left[\operatorname{eval}\left(C_{1},\{f\}\right) \wedge \operatorname{eval}\left(C_{2},\{t\}\right)\right] \overline{\operatorname{start}_{\text {Act }_{2}}}\{(++,}
\end{aligned}
$$

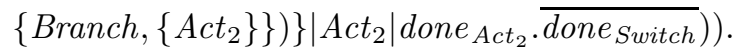

5) Formalism of the Pick Structure. The Pick structure defines execution selection among different services and structures in a grid workflow based on message triggers:

$$
\begin{aligned}
& \operatorname{Pick}\left(f_{s d}\left(A c t_{1}\right), f n_{s d}\left(A_{c t}\right), \text { port }_{p 1}, \text { port }_{p 2}\right. \text {, timeout, } \\
& \text { start } \left._{\text {Pick }}, \text { done }_{\text {Pick }}\right) \\
& =_{\text {def }}\left(\text { new } \text { start }_{\text {Act }_{1}} \text { start }_{\text {Act }_{2}} \text { done }_{\text {Act }_{1}} \text { done }_{\text {Act }_{2}}\right. \text { ) } \\
& \text { start }_{\text {Pick }} \cdot\left(\text { port }_{p 1}\left\{++,\left\{\text { msgPort }_{1},\left\{\text { port }_{p 1}\right\}\right\}\right\}\right. \text {. } \\
& \text { start }_{\text {Act }_{1}}\left\{\left(++,\left\{\text { Event },\left\{\text { Act }_{1}\right\}\right\}\right),(-- \text {, }\right. \\
& \left.\left.\left\{\text { msgPort }_{1},\left\{\text { port }_{p 1}\right\}\right\}\right)\right\} \mid \text { Act }_{1} \mid \text { done }_{\text {Act }_{1}} \text {. } \\
& \overline{\text { done } \text { Pick }}+ \\
& \text { port }_{p 2}\left\{++,\left\{\text { msgPort }_{2},\left\{\text { port }_{p 2}\right\}\right\}\right\} \text {. } \\
& \text { start }_{\text {Act }_{2}}\left\{\left(++,\left\{\text { Event },\left\{\text { Act }_{2}\right\}\right\}\right),(-- \text {, }\right. \\
& \left.\left.\left\{\text { msgPort }_{2},\left\{\text { port }_{p 2}\right\}\right\}\right)\right\} \mid \text { Act }_{2} \mid \text { done }_{\text {Act }_{2}} \text {. } \\
& \left.\overline{\text { done }_{\text {Pick }}}\right)+ \\
& \text { timeout } \left.\{(++,\{\text { Event },\{\text { Timeout }\}\})\} . \overline{\text { done }_{\text {Pick }}}\right) \text {. }
\end{aligned}
$$

6) Formalism of the Link Structure. The Link structure imposes synchronization constraints on activities in a grid workflow. Each Link has a source and a target activity, which restricts that the target activity can only be executed after the source activity is done.

$$
\operatorname{Link}_{i}\left(\text { done }_{\text {in }}, \text { neg }_{\text {in }}, \text { ack, nack }\right)
$$

$={ }_{\mathrm{def}}$ EvalTransCondition $_{i}\left(\right.$ done $_{i n}$, neg $_{\text {in }}$, ack, nack

$={ }_{\mathrm{def}}$ done $_{\text {in }} \cdot \overline{a c k}+n e g_{\text {in }} \cdot \overline{\text { nack }}, \quad i=1, \ldots, n$,

Links $\left(\right.$ done $_{\text {in }}$, neg $_{\text {in }}$, done $_{\text {links }}$, deathpath $)$ $={ }_{\operatorname{def}}($ new ack nack $)\left(\operatorname{Link}_{1} \mid \cdots\right.$

\author{
$\left|\operatorname{Link}_{n}\right| \underbrace{\operatorname{ack} \cdot(\ldots . .(a c k}_{n} \cdot \overline{\text { done }_{\text {links }}}+$

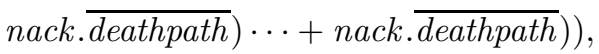 \\ ActWithLinks (freeN) \\ $={ }_{\text {def }}$ start. (done links $_{\text {. new }} t f(\overline{\text { evaljoin }}\langle t, f\rangle$. \\ $\left(t .\left(\right.\right.$ new start $_{\text {Act }}$ done $\left._{\text {Act }}\right)\left(\overline{\text { start }_{\text {Act }}} \mid\right.$ Act $\mid$ \\ done $\left._{\text {Act }} . \overline{\text { done }} . \Pi \overline{\text { done }_{\text {out }}}\right)+$ \\ $f\{++,\{$ Exception, $\{$ Act $\}\}\}$. \\ $\overline{\text { throw }}($ joinfailure $\rangle))+$ deathpath. $\left.\Pi \overline{n^{2} g_{\text {out }}}\right)$ \\ free $N={ }_{\text {def }}\left\{\right.$ start, done, done $_{\text {links }}$, done $_{\text {out }}$, neg $_{\text {out }}$, \\ deathpath, evaljoin, fault, joinfailure, $f n_{s d}($ Act $\left.)\right\}$.
}

\section{Grid Workflows and Standard Regions}

\subsection{Preliminary Constraints}

Considering that there are various grid workflow specification languages, common notations used in this paper are provided in Fig.1 to visually represent a grid workflow model. Modeling elements in Fig.1 cover as many existing workflow languages (e.g., BPEL4WS) as possible.

To prevent possible construction of unstructured grid workflows, syntactical constraints are defined as a unified basis for our region analysis, which is concluded from soundness criteria (no deadlocks and no multiple service activity instances on the same service activity) ${ }^{[24]}$.

Constraint 1. We refer a Srv\&Ctrl node to a Grid Service Activity, Subflow or Control node and refer a SrvFlow node to a Srv\&Ctrl or Data Service node.

Constraint 2. Each grid workflow has exactly one explicit Begin node and End node (which will be later relaxed in our $R R A$ approach).

Constraint 3. Every Srv\&Ctrl node must be syntactically reachable from the Begin node and can reach the End node by transitions (i.e., no dangling grid service activity, subflow, or control nodes).

Constraint 4. Each transition has exactly one source/target Srv\&Ctrl node. Each data channel has at

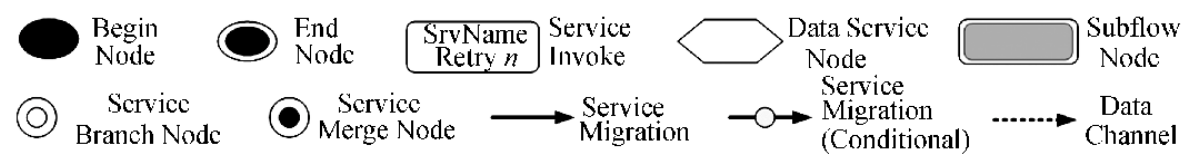

(a)

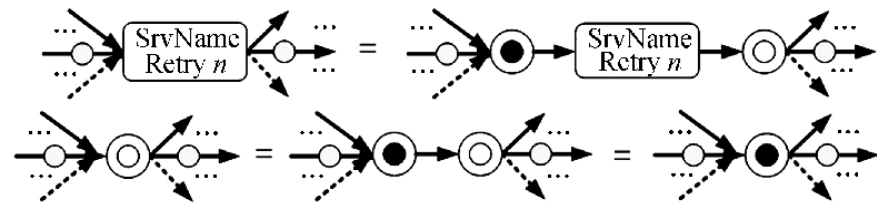

(b)

Fig.1. Notations. (a) Visual notations of grid workflow elements. (b) Annotation shortcuts for grid workflow controls. 
most one source/target SrvFlow node (with one of them must be a data service Node). Therefore, a Data Channel is called fluent if and only if (IFF) its source/target Data Service node is reachable from the Begin node or can reach the End node.

Constraint 5. Multiple inputs and outputs are allowed for a Grid Service Activity and Control node. Their equivalent semantics are illustrated in Fig.1(b).

Constraint 6. Arbitrary cycles are allowed as long as no unstructured workflow models are caused.

Fig.2 illustrates an example gravitational wave data analysis workflow $S F 1$ based on visual notations provided in Fig.1.

\subsection{Standard Regions}

The most common temporal relations in formal verification is the combinations of "what will eventually/always happen in the future" and "something will hold until an event is received". Therefore the idea is to encapsulate a grid workflow into separate sub workflows such that important relations can be directly implied between them. Consequently, instead of global reasoning of the whole workflow, which is quite costly, it can be equivalent to locally investigate behaviors of sub workflows.

Denote $N_{1} \rightarrow N_{2} \rightarrow \cdots \rightarrow N_{m}$ to be a directed path from node $N_{1}$ to $N_{m}$ in a grid workflow. Note that by $N_{1} \rightarrow N_{2}$, it only means that $N_{1}$ and $N_{2}$ are syntactically connected by transitions or data channels and $N_{2} / N_{1}$ is the target/source of the transition or data channel. Consequently the structural information of a workflow is our primary concern in the definition of regions.

Definition 1 (Region). Two Srv\&Ctrl nodes or Begin/End nodes Nhead and Ntail form a region in a grid workflow $\Gamma$, denoted by $\left.\left\{N_{\text {head }}, N_{\text {tail }}\right\}, I F F: 1\right)$ $\nexists N_{\text {head }} \rightarrow N_{1} \rightarrow \cdots \rightarrow N_{m} \rightarrow$ End where End is the End node of $\Gamma$, and $N_{i}$ ! $\left.=N_{\text {tail }}(i=1, \ldots, m) ; 2\right) A$ Begin $\rightarrow N_{1} \rightarrow \cdots \rightarrow N_{m} \rightarrow N_{\text {tail }}$, where Begin is the Begin node of $\Gamma$, and $N_{i} !=N_{\text {head }}(i=1, \ldots, m)$.

More intuitively, a region $\left\{N_{\text {head }}, N_{\text {tail }}\right\}$ specifies a structure in which node $N_{\text {head }}$ will always reach $N_{\text {tail }}$ in order for it to reach the End node in $\Gamma$ (and vice versa). For example, in Fig.2, \{TrigBank_H2_3, thIncall_L1H2\} is a region while $\left\{s I n c a_{-} L 1 H 1\right.$, thIncall_L $\left.L 1 H 2\right\}$ is not. The whole grid workflow $\Gamma$ itself also forms a region. A node $N^{\prime}$ is thus said to be within a region $\left\{N_{1}, N_{2}\right\}$ (denote by $N^{\prime} \subset\left\{N_{1}, N_{2}\right\}$ ) if there exists a path $N_{1} \rightarrow \cdots \rightarrow N^{\prime} \rightarrow \cdots \rightarrow N_{2}$. The definition of region preserves the following property.

Proposition 1. If $\left\{N_{1}, N_{2}\right\}$ and $\left\{N_{2}, N_{3}\right\}$ are two regions in $\Gamma$ where $N_{1}, N_{2}, N_{3}$ are uniquely identified nodes, $\left\{N_{1}, N_{3}\right\}$ also forms a region in $\Gamma$.

To well control the number of regions that can be identified in a grid workflow and to make them cover the whole grid workflow, it is required to further define a standard granularity for regions.

Definition 2 (Maximized Region). Two Srv\&Ctrl nodes or Begin/End nodes $N_{\text {head }}$ and $N_{\text {tail }}$ form a maximized region in a grid workflow $\Gamma$, IFF $\forall$ Begin $\rightarrow N_{1} \rightarrow \cdots \rightarrow N_{m} \rightarrow$ End where Begin, End are the Begin node and the End node of $\Gamma, N_{\text {head }}$ and $N_{\text {tail }}$ are contained in the path and $N_{\text {head }} \neq N_{\text {tail }}$.

It can be seen from the definition that a maximized region is also a region, and thus satisfies Proposition 1.

Definition 3 (Decomposition). A maximized region $\left\{N_{1}, N_{2}\right\}$ in $\Gamma$ is said to be decomposable IFF: $1)$ there are more than one path $N_{1} \rightarrow \cdots \rightarrow N_{2}$ s.t. $N_{1}$ can reach $N_{2}$ in $\Gamma$ and $\exists N^{\prime} \subset\left\{N_{1}, N_{2}\right\}$, s.t. $\left\{N_{1}, N^{\prime}\right\}$ or $\left\{N^{\prime}, N_{2}\right\}$ is a maximized region; or 2$)$ there is only one path $N_{1} \rightarrow \cdots \rightarrow N^{\prime} \rightarrow \cdots \rightarrow N_{2}$ s.t. $N_{1}$ can reach $N_{2}$ in $\Gamma$ and for any $N_{0} \rightarrow N_{1}$ and $N_{2} \rightarrow N_{3}$, either $\left\{N_{0}, N_{2}\right\},\left\{N_{1}, N_{3}\right\}$ or $\left\{N_{0}, N_{3}\right\}$ is a maximized region. Moreover, for nodes $N_{1}^{\prime}, \ldots, N_{m}^{\prime}$ within region $\left\{N_{1}, N_{2}\right\}$, the set of maximized regions $\left\{\left\{N^{\prime}, N^{\prime \prime}\right\} \mid\left\{N^{\prime}, N^{\prime \prime}\right\}=\left\{N_{1}, N_{1}^{\prime}\right\}\right.$ or $\left\{N_{1}^{\prime}, N_{2}^{\prime}\right\}$ or $\ldots$ or $\left.\left\{N_{m}^{\prime}, N_{2}\right\}\right\}$ is said to be a total decomposition of $\left\{N_{1}, N_{2}\right\}$ IFF all $\left\{N^{\prime}, N^{\prime \prime}\right\}$ s are maximized regions and are not further decomposable.

Definition 4 (Standard Region). A maximized region $\left\{N_{1}, N_{2}\right\}$ in $\Gamma$ is a standard region IFF $\left\{N_{1}, N_{2}\right\}$ belongs to the total decomposition of $\Gamma$.

Since $\Gamma$ itself also forms a maximized region, Definitions 2 and 3 imply that a standard region will always exist for $\Gamma$ (in the worst case the only standard region will be $\Gamma$ itself). For example in Fig.2, while $\{$ TrigBank_H2_3, thIncall_L $L 1 H 2\}$ is a region, it is neither a maximized region nor a standard region.

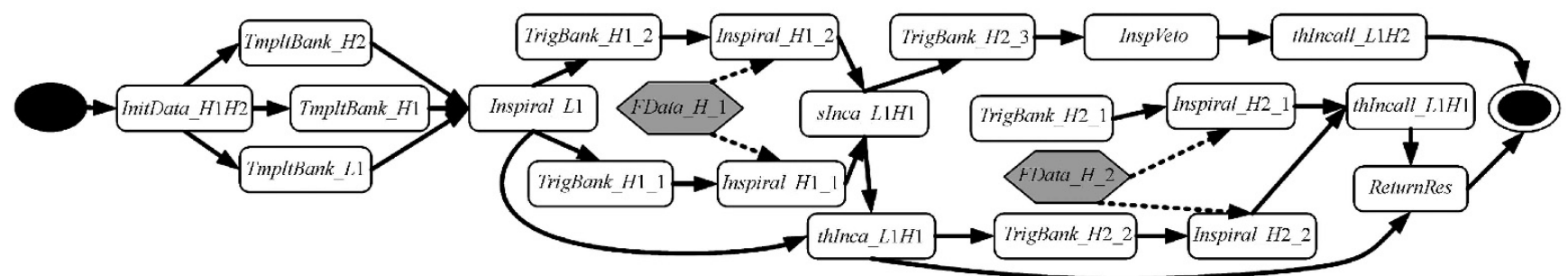

Fig.2. Gravitational wave data analysis - case study $1(S F 1)$. 
However, $\{$ Begin, Inspiral $\}$ is a standard region for $\Gamma$. As the following proposition states, standard regions enjoy important sequential relations between each other that can be used for verification decomposition.

Proposition 2. For any two different standard regions $\left\{N_{1}^{\prime}, N_{2}^{\prime}\right\}$ and $\left\{N_{1}^{\prime \prime}, N_{2}^{\prime \prime}\right\}$ in $\Gamma$, one of the following two temporal relations can be held:

1) Any Srv\&Ctrl node $N^{\prime \prime}$ in $\left\{N_{1}^{\prime \prime}, N_{2}^{\prime \prime}\right\}$ (including $N_{1}^{\prime \prime}$ and $N_{2}^{\prime \prime}$ ) is preceded by any Srv\&Ctrl node $N^{\prime}$ in $\left\{N_{1}^{\prime}, N_{2}^{\prime}\right\}$ (including $N_{1}^{\prime}$ and $N_{2}^{\prime}$ ), in that $N^{\prime} \rightarrow \cdots \rightarrow$ $N^{\prime \prime}$ always exists with zero or multiple transitions/data channels in all paths in $\Gamma$;

2) Any Srv\&Ctrl node $N^{\prime}$ in $\left\{N_{1}^{\prime}, N_{2}^{\prime}\right\}$ (including $N_{1}^{\prime}$ and $N_{2}^{\prime}$ ) is preceded by any Srv\&Ctrl node $N^{\prime \prime}$ in $\left\{N_{1}^{\prime \prime}\right.$, $\left.N_{2}^{\prime \prime}\right\}$ (including $N_{1}^{\prime \prime}$ and $N_{2}^{\prime}$ ), in that $N^{\prime \prime} \rightarrow \cdots \rightarrow N^{\prime}$ always exists with 0 or multiple transitions/data channels in all paths in $\Gamma$.

The proposition indicates that strict precedence relations are preserved between standard regions. Accordingly the corresponding algorithm (TotalDecomposition) for decomposing a grid workflow into its standard regions is also implemented in this work. Fig.2 also shows the result of standard regions in the given case study of gravitational wave data analysis.

\section{Verification Decomposition based on Standard Regions}

Apart from the decomposition of grid workflows, the decomposition of corresponding formal verification strategies are also developed, which include:

1) how to exploit the properties of a standard region into its verification;

2) how to exploit local verification of a standard region into verification of other standard regions;

3) how to deduct the global verification result based on local verification of standard regions.

Above issues can be actually transformed into a special modular model checking problem ${ }^{[25]}$. As we know, the idea of formal verification is to find all states $\{s \in M \mid M, s \models f\}$, where $M$ is the state model ${ }^{[13]}$ (e.g., kripke structure, automata, etc.) of the target system to be verified and $f$ is the desired property. It is said that $M$ satisfies $f$ (i.e., $M \models f$ ) if the set of states $s$ is not empty. A modular model checking tries to deduct the formal verification procedure in the following form:

$$
\frac{\langle T R U E\rangle M\langle\varphi\rangle \quad\langle\varphi\rangle M^{\prime}\langle\psi\rangle}{\langle T R U E\rangle M \mid M^{\prime}\langle\psi\rangle} .
$$

The deduction tries to prove that if model $M$ satisfies property $\varphi(\langle T R U E\rangle M\langle\varphi\rangle)$ and model $M^{\prime}$ satisfies property $\psi$ under the assumption that its environment satisfies property $\varphi\left(\langle\varphi\rangle M^{\prime}\langle\psi\rangle\right)$, the parallel composition of $\left(M \mid M^{\prime}\right)$ will satisfy property $\left(\langle T R U E\rangle M \mid M^{\prime}\langle\psi\rangle\right)$. An essential procedure in the above deduction is how to define and implement $\langle\varphi\rangle M^{\prime}\langle\psi\rangle$ such that the deduction will hold true. Consequently, our decomposition strategy of verifications based on standard regions follows the idea below: given the total decomposition $\left\{M_{1}, M_{2}, \ldots, M_{n}\right\}$ of a grid workflow $\Gamma$ where $M_{i}=\left\{N_{i}, N_{i+1}\right\}, N_{i}, N_{i+1} \in \Gamma$, the verification of a desired property $\psi$ is carried out on $M_{n}, \ldots, M_{1}$ separately, whereas the verification of $M_{i}$ against $\psi$ will be based on the satisfaction of $M_{i+1} ; \ldots ; M_{n}$ against $\psi$ such that the satisfaction of the complete workflow $\Gamma$ against $\psi$ can be eventually deducted.

$$
\frac{\langle T R U E\rangle M_{i+1} ; \cdots ; M_{n}\left\langle\psi_{i+1}\right\rangle \quad\left\langle\psi_{i+1}\right\rangle M_{i}\left\langle\psi_{i}\right\rangle}{\langle T R U E\rangle M_{i} ; M_{i+1} ; \cdots ; M_{n}\left\langle\psi_{i}\right\rangle} .
$$

Here we have $1 \leqslant i \leqslant n-1$ and $M ; M^{\prime}$ indicates the sequential composition of the identified standard regions since sequential relations are preserved among standard regions. The following takes LTL-X (a popular temporal logic with universal path qualifiers and no next operators) ${ }^{[13]}$ as the target for the implementation of $\langle\varphi\rangle M^{\prime}\langle\psi\rangle$ (i.e., both $\varphi$ and $\psi$ are specified in LTL$\mathrm{X})$. LTL-X is an intuitive and shuttering closed logic with wide formal verification tool support. Since an important theoretical foundation is that LTL-X formulae can be transformed to equivalent generalized büchi automaton $^{[13]},\langle\varphi\rangle M^{\prime}\langle\psi\rangle$ can be obtained by verifying $\operatorname{Trans}(\varphi) \mid M^{\prime} \models \psi$, where $\operatorname{Trans}(\varphi)$ indicates the equivalent automata for $\varphi$. However in this work, the sequential nature of standard regions enables us to further avoid the cost for automata composition. The following provides the implementation of $\langle\varphi\rangle M^{\prime}\langle\psi\rangle$ based on the semantics of LTL-X formulae on system states and paths.

Definition 5 (Association States). Given the total decomposition $\left\{M_{1}, M_{2}, \ldots, M_{n}\right\}$ of a grid workflow $\Gamma$ where $M_{i}=\left\{N_{i}, N_{i+1}\right\}, N_{i}, N_{i+1} \in \Gamma$, denote TransSys $(\Gamma, \Phi)$ to be the automata for $\Gamma$ under the given initial state set of $\Phi$. Since $M_{i}$ and $M_{i+1}$ share the service node $N_{i+1}$, the set of association states $\operatorname{Im}\left(M_{i}, M_{i+1}, \Gamma\right)$ is the states when $M_{i} ; M_{i} ; \ldots ; M_{n}$ transits to the process of $M_{i+1} ; M_{i+2} ; \cdots ; M_{n}$.

The association states literally indicate the region initial states for the previous local verification

$$
\left(\langle T R U E\rangle M_{i+1} ; M_{i+2} ; \cdots ; M_{n}\left\langle\psi_{i+1}\right\rangle\right)
$$

and the region ending states for the current local verification $\left(\left\langle\psi_{i+1}\right\rangle M_{i}\left\langle\psi_{i}\right\rangle\right)$ in the deduction procedure (3).

Definition 6 (Region Initial/Ending States). Given the total decomposition $\left\{M_{1}, M_{2}, \ldots, M_{n}\right\}$ of a grid workflow $\Gamma$ where $M_{i}=\left\{N_{i}, N_{i+1}\right\}, N_{i}$, 
$N_{i+1} \in \Gamma$, the region ending states for $M_{i}\left(\mathbb{E}\left(M_{i}\right)\right)$ and the region initial states for $M_{i+1} ; M_{i+2} ; \cdots ; M_{n}$ $\left(\mathbb{S}\left(M_{i+1} ; M_{i+2} ; \cdots ; M_{n}\right)\right)$ are both $\operatorname{Im}\left(M_{i}, M_{i+1}, \Gamma\right)$.

Lemma 1. $\forall$ state $s_{i} \in \operatorname{Trans}\left(M_{i}, \mathbb{S}\left(M_{i} ; \ldots ; M_{n}\right)\right)$ and $s_{i+1} \in \operatorname{Trans}\left(M_{i+1}, \mathbb{S}\left(M_{i+1} ; \ldots ; M_{n}\right)\right)$, if $s_{i}=$ $s_{i+1}$, then $s_{i}, s_{i+1} \in \operatorname{Im}\left(M_{i}, M_{i+1}, \Gamma\right)$.

The lemma states that in the total decomposition of $\Gamma$, the only shared states of the corresponding automata for standard regions $M_{i}$ and $M_{i+1}$ are their association states. This implies that no states in one standard region will loop back to states in another standard region, which is a direct result of Definition 3 and Constraint 6 .

Proposition 3. Given the total decomposition $\left\{M_{1}, M_{2}, \ldots, M_{n}\right\}$ of grid workflow $\Gamma$, the desired formula $\Psi$ and all of its sub-formulae $\varphi \in \operatorname{sub}(\Psi)$, based on the result that

$$
\operatorname{Trans}\left(M_{i+1} ; M_{i+2} ; \ldots ; M_{n}, \mathbb{S}\left(M_{i+1} ; \ldots ; M_{n}\right)\right) \models \varphi
$$

(i.e., $\left.\langle T R U E\rangle M_{i+1} ; M_{i+2} ; \ldots ; M_{n}\langle\varphi\rangle\right)$, it can be deduced that

$$
\langle T R U E\rangle M_{i} ; M_{i+1} ; M_{i+2} ; \ldots ; M_{n}\langle\varphi\rangle
$$

also holds if:

- If $\varphi=p$ where $p$ is an atomic Boolean proposition, $\langle\varphi\rangle M_{i}\langle p\rangle$ holds IFF $M_{i}, \mathbb{S}\left(M_{i} ; \ldots ; M_{n}\right) \models p ;$

- If $\varphi=\varphi_{1} \vee \varphi_{2},\langle\varphi\rangle M_{i}\left\langle\varphi_{1} \vee \varphi_{2}\right\rangle$ holds IFF $M_{i}$, $\mathbb{S}\left(M_{i} ; \ldots ; M_{n}\right) \models \varphi_{1}$ or $M_{i}, \mathbb{S}\left(M_{i} ; \ldots ; M_{n}\right) \models \varphi_{2} ;$

- If $\varphi=\neg \varphi_{1}, \quad\langle\varphi\rangle M_{i}\left\langle\neg \varphi_{1}\right\rangle$ holds IFF $M_{i}$, $\mathbb{S}\left(M_{i} ; \ldots ; M_{n}\right) \not \models \varphi_{1}$

- If $\varphi=\varphi_{1} \cup \varphi_{2},\langle\varphi\rangle M_{i}\left\langle\varphi_{1} \mathrm{U} \varphi_{2}\right\rangle$ holds IFF for any state sequence starting from $\mathbb{S}\left(M_{i} ; \ldots ; M_{n}\right): \pi=$ $\mathbb{S}\left(M_{i} ; \ldots ; M_{n}\right), \sigma_{1}, \sigma_{2}, \ldots, \sigma_{k}$, it has:

$\circ \exists i \in N^{+}$, s.t. $\sigma_{i} \in \pi$ and $\sigma_{i} \in \mathbb{S}\left(M_{i+1} ; \ldots ; M_{n}\right)$, $\left(M_{i+1} ; \ldots ; M_{n}, \sigma_{i}\right) \models \varphi_{1} U \varphi_{2} . \quad \forall$ infinite state sequence $\pi^{\prime} \in \operatorname{Trans}\left(\varphi_{1} U \varphi_{2}\right)=\sigma^{0}, \sigma^{1}, \sigma^{2}, \ldots$, for each such $\sigma_{i}$ and $\pi^{\prime \prime}=\mathbb{S}\left(M_{i} ; \ldots ; M_{n}\right), \sigma_{1}, \ldots, \sigma_{i}$, $\sigma^{1}, \sigma^{2} \ldots,\left(M_{i}, \pi^{\prime \prime}\right) \models \varphi_{1} U \varphi_{2}$ holds true; otherwise:

○ $\left(M_{i}, \pi^{*}\right) \models \varphi_{1} U \varphi_{2}$, where $\pi^{*}=\mathbb{S}\left(M_{i} ; \ldots ; M_{n}\right)$, $\sigma_{1}, \sigma_{2}, \ldots, \sigma_{k}, \ldots$;

- If $\varphi=G \varphi_{1},\langle\varphi\rangle M_{i}\left\langle G \varphi_{1}\right\rangle$ holds IFF for any state sequence starting from $\mathbb{S}\left(M_{i} ; \ldots ; M_{n}\right): \pi=$ $\mathbb{S}\left(M_{i} ; \ldots ; M_{n}\right), \sigma_{1}, \sigma_{2}, \ldots, \sigma_{k}$, it has:

$\circ \exists i \in N^{+}$, s.t. $\sigma_{i} \in \pi$ and $\sigma_{i} \in \mathbb{S}\left(M_{i+1} ; \ldots ; M_{n}\right)$, $\left(M_{i+1} ; \ldots ; M_{n}, \sigma_{i}\right) \models G \varphi_{1} . \forall$ infinite state sequence $\pi^{\prime} \in \operatorname{Trans}\left(\varphi_{1} U \varphi_{2}\right)=\sigma^{0}, \sigma^{1}, \sigma^{2}, \ldots$, for each such $\sigma_{i}$ and $\pi^{\prime \prime}=\mathbb{S}\left(M_{i} ; \ldots ; M_{n}\right), \sigma_{1}, \ldots, \sigma_{i}, \sigma^{1}, \sigma^{2}, \ldots$, $\left(M_{i}, \pi^{\prime \prime}\right) \models G \varphi_{1}$ holds true; otherwise:

$\circ\left(M_{i}, \pi^{*}\right) \models G \varphi_{1}$, where $\pi^{*}=\mathbb{S}\left(M_{i} ; \ldots ; M_{n}\right)$, $\sigma_{1}, \sigma_{2}, \ldots \sigma_{k}, \ldots$

- If $\varphi=\varphi_{1} W \varphi_{2}, \quad\langle\varphi\rangle M_{i}\left\langle\varphi_{1} W \varphi_{2}\right\rangle$ holds IFF $\langle\varphi\rangle M_{i}\left\langle G \varphi_{1} \vee\left(\varphi_{1} U \varphi_{2}\right)\right\rangle$;

- If $\varphi=\varphi_{1} R \varphi_{2}, \quad\langle\varphi\rangle M_{i}\left\langle\varphi_{1} R \varphi_{2}\right\rangle$ holds IFF $\langle\varphi\rangle M_{i}\left\langle G \varphi_{2} \vee\left(\varphi_{2} U\left(\varphi \wedge \varphi_{1}\right)\right)\right\rangle 1$;
- If $\varphi=F \varphi_{1},\langle\varphi\rangle M_{i}\left\langle F \varphi_{1}\right\rangle$ holds IFF $\langle\varphi\rangle M_{i}$ $\left.\left\langle T R U E U \varphi_{1}\right)\right\rangle$.

Proposition 3 implies an important decomposition strategy for formal verification based on standard regions. That is, given a standard region $M_{i}$ in a grid workflow $\Gamma$, the desired LTL-X formula $\Psi$ and its sub formulae $\varphi \in \operatorname{sub}(\Psi)$, if $\langle T R U E\rangle M_{i+1} ; M_{i+2} ; \ldots ; M_{n}\langle\varphi\rangle$ holds, we can deduct the satisfaction of $\langle T R U E\rangle M_{i} ; M_{i+1} ; M_{i+2} ; \ldots ; M_{n}\langle\varphi\rangle$ by investigating whether $\left(\operatorname{Trans}\left(M_{i}, \mathbb{S}\left(M_{i} ; \ldots ; M_{n}\right)\right)\right.$; $\operatorname{Trans}(\varphi)), \mathbb{S}\left(M_{i} ; \ldots ; M_{n}\right) \models \varphi$ holds. Here ";" represents the sequential composition of $\operatorname{Trans}\left(M_{i}\right.$, $\left.\mathbb{S}\left(M_{i} ; \ldots ; M_{n}\right)\right)$ and $\operatorname{Trans}(\varphi)$ in order to construct the paths of $\mathbb{S}\left(M_{i} ; \ldots ; M_{n}\right), \sigma_{1}, \ldots, \sigma_{i}, \sigma^{1}, \sigma^{2}, \ldots$

\section{Grid Workflow Decomposition Using Relaxed Region Analysis}

In above sections, a complete method for formal verification of grid workflows by decomposing both the workflow and the corresponding verification strategy is formulated. However, one deficiency of the above workflow decomposition is that it has imposed strong constraints (see Section 2) on grid workflow structure analysis, which sometimes limits the improvement of the verification performance because the identified standard regions are not small enough. For example, in the decomposition result in Fig.2, the identified standard region $\{$ Inspiral_L1, End $\}$ can be still considered as a complex sub workflow compared to the complete grid workflow.

It is found that one of the key factors in decomposing the verification of a grid workflow $\Gamma$ is to assure that

$\operatorname{TransSys}\left(M_{i}, \mathbb{S}\left(M_{i} ; \ldots ; M_{n}\right)\right) \supseteq \mathbb{S}\left(M_{i+1} ; M_{i+2} ; \ldots ; M_{n}\right)$

s.t. the sequential composition of $M_{i} ; \ldots ; M_{n}$ will not loose complete behaviors in the original grid workflow. Under this condition, it is inspired to relax Constraint 2 in Subsection 3.1 to allow multiple End nodes in $\Gamma$ such that potential parallel branches can also be discovered in addition to the sequential standard regions.

Relaxation of Constraint 2. Each grid workflow has exactly one explicit Begin node and can be relaxed to allow multiple End nodes. New End nodes after relation are named secondary end nodes $\left(V_{E n d}\right)$.

For a standard region, denote $M_{i} /\left(N_{1} \rightarrow \cdots \rightarrow N_{m}\right)$ $\left(N_{j} \in M_{i}, j=1, \ldots, m\right)$ as the operation of removing a branch in $\Gamma$ with corresponding grid workflow nodes, transitions and data channels. It is then expected to find more potential standard regions in a grid workflow by temporarily removing a selected branch, and to make the verification decomposition result still work in this relaxed context. 
Definition 7 (Parallel Branch). In the total decomposition $\left\{M_{1}, M_{2}, \ldots, M_{n}\right\}$ of a grid workflow $\Gamma$, a connected path $\mathbb{C P}=N_{1} \rightarrow^{*} N_{2} \rightarrow \cdots \rightarrow V_{\text {End }}$ in $M_{n}$ is called a parallel branch, IFF: 1) $\rightarrow^{*}$ is a transition with no condition; 2$)$ in the total decomposition $\left\{M_{1}^{\prime}, M_{2}^{\prime}, \ldots, M_{m}^{\prime}\right\}$ of $M_{n} /\left(N_{2} \rightarrow \cdots \rightarrow V_{E n d}\right)$, if $N_{1} \in M_{i}^{\prime}(i=1,2, \ldots, m), \forall i<j \leqslant m, \exists$ path $N_{j_{1}} \rightarrow \cdots \rightarrow N_{j k}$ in $M_{j}$, s.t. $\exists N_{j k} \in N_{2} \rightarrow \cdots \rightarrow$ $V_{\text {End }}$; 3) $\nexists$ other path $N_{0} \rightarrow^{*} N_{1} \rightarrow N_{2} \rightarrow \cdots \rightarrow V_{\text {End }}$ in $M_{i}^{\prime}$ s.t. it also satisfies 2 ).

The above definition of a parallel branch $\mathbb{C P}=$ $N_{1} \rightarrow^{*} N_{2} \rightarrow \cdots \rightarrow V_{E n d}$ indicates a path that ends with $V_{E n d}$, such that a parallel composition relation holds between grid service nodes in $N_{2} \rightarrow \cdots \rightarrow V_{E n d}$ and new discovered standard regions after node $N_{1}$ (i.e., there are no control/data constraints in service execution among them). In the total decomposition $\left\{M_{1}^{\prime}, M_{2}^{\prime}, \ldots, M_{m}^{\prime}\right\}$ of $M_{n} /\left(N_{2} \rightarrow \cdots \rightarrow V_{E n d}\right)$, if $N_{1} \in M_{i}^{\prime}$, it is called that the parallel branch $\mathbb{C P}$ belongs to $M_{i}^{\prime}$, denoted by $M_{i}^{\prime}(\mathbb{C P}) . \quad\left\{M_{1}, M_{2}, \ldots, M_{1}^{\prime}\right.$, $\left.M_{2}^{\prime}, \ldots, M_{i}^{\prime}(\mathbb{C P}), \ldots, M_{m}^{\prime}\right\}$ is therefore called the relaxed total decomposition after the relaxation of $\mathbb{C P}=$ $N_{1} \rightarrow N_{2} \rightarrow \cdots \rightarrow V_{E n d}$ for grid workflow $\Gamma$.

Denote $\mathbb{C P}^{\prime}=N_{2} \rightarrow \cdots \rightarrow V_{\text {End }}$, because $\mathbb{C P}^{\prime}$ forms a parallel relation with all the rest standard regions $\left(M_{i+1}^{\prime}, \ldots, M_{m}^{\prime}\right)$ when $M_{i}^{\prime}(\mathbb{C P})$, we have $\operatorname{Trans}\left(\left(M_{k}^{\prime}, \ldots, M_{m}^{\prime} \mid \mathbb{C P}^{\prime}\right)\right) \supseteq \operatorname{Trans}\left(\left(M_{k+1}^{\prime}, \ldots\right.\right.$, $\left.\left.M_{m}^{\prime} \mid \mathbb{C P}^{\prime}\right)\right)$ for any $i \leqslant k<m$ under the same initial state Init. Therefore the verification under relaxed region analysis can also reuse the results in Section 4. The whole deduction procedure includes four steps, as shown in (3)

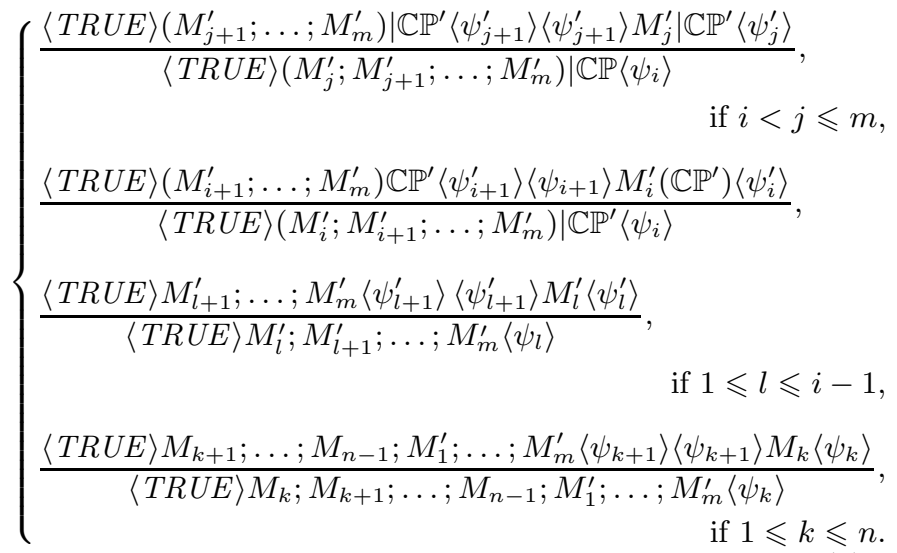

The former two in (3) represent the cases of standard regions with consideration of parallel branches, while the latter two are used to deal with normal situations described in Section 4. The RRA flowchart is illustrated in Fig.3, which is based on the TotalDecomposition algorithm.

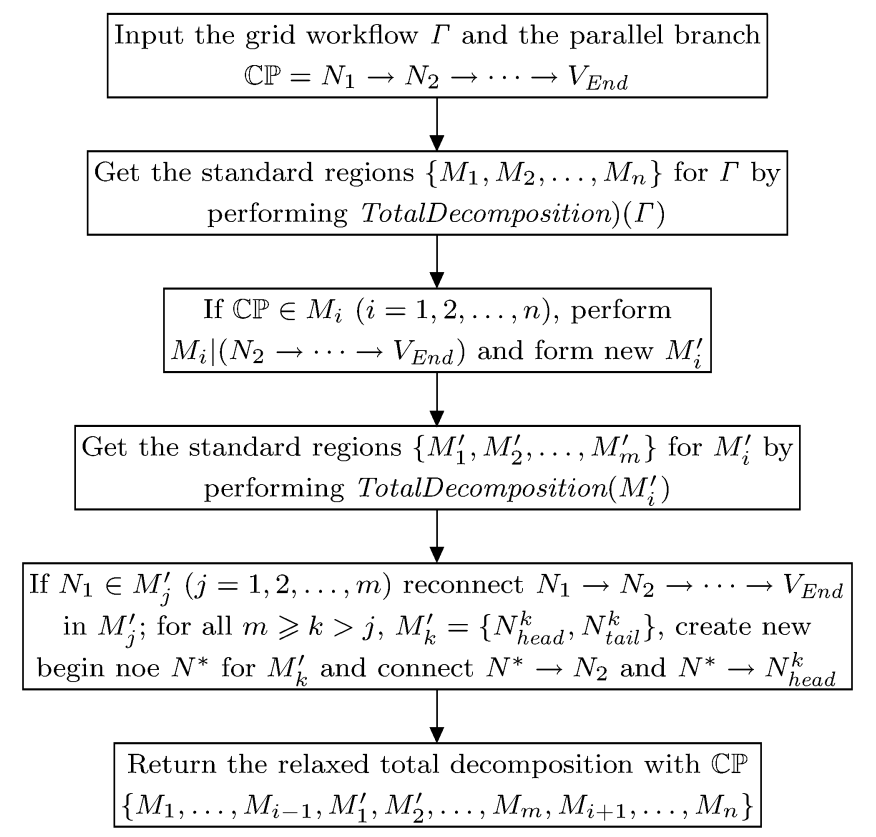

Fig.3. RRA flow chart.

In the gravitational wave workflow $S F 1$, since sInca_L1H1 $\rightarrow^{*}$ TrigBank_H2_3 $\rightarrow$ InspVeto $\rightarrow$ thIn call_L1H2 $\rightarrow V_{E n d}$ is a parallel branch, the original standard region $\{$ Inspiral_L1, End $\}$ can be further decomposed into two smaller regions, $\{$ Inspiral_L1, thInca_L1H1\} and $\{$ thInca_L1H1, End $\}$, based on the RRA approach as described in Fig.4.

\section{System Implementation and Performance Evaluation}

\subsection{GridPiAnalyzer}

State Pi calculus is used in our experiments for grid workflow modeling. The RRA approach with the verification strategy in Section 3 and Section 4 is implemented in our GridPiAnalyzer to further improve its performance in reasoning grid workflows. GridPiAnalyzer is an automatic analyzer designed for ensuring reliability of grid workflow based on its Pi calculus formalism and verification.

GridPiAnalyzer accepts target grid workflow scripts, e.g., DAG specifications, BPEL4WS and the Unified Modeling Language (UML) 2.0 activity diagrams. It automatically transforms grid workflow specifications into the process algebra of state Pi calculus and deduces the result into labeled transition systems according to the operational semantics of state Pi calculus. A visual environment is provided for specifying required temporal properties on grid workflows using LTL (Linear Temporal Logic). These formulae, together with the transition system, are accepted to perform the formal verification. 


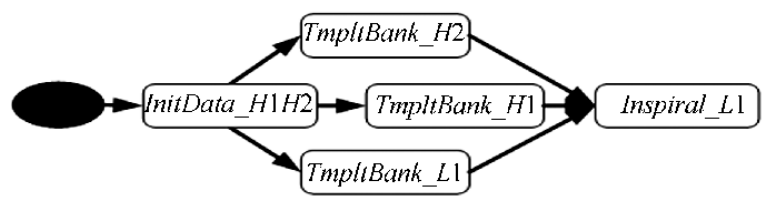

(a)

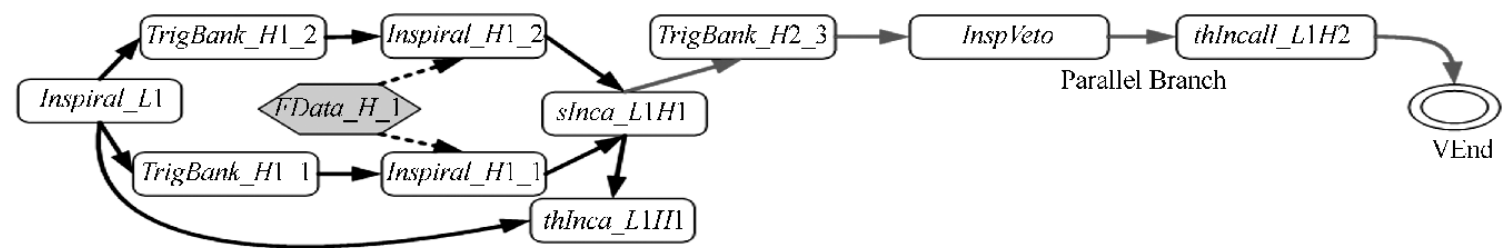

(b)

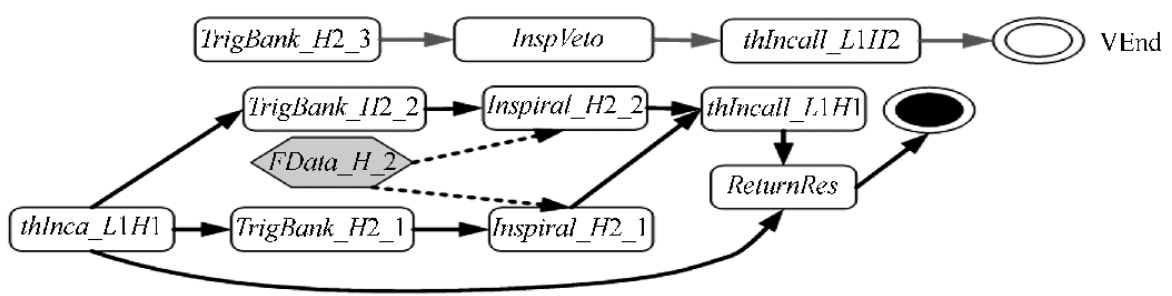

(c)

Fig.4. Relaxed region decomposition for $S F 1$. (a) $R_{11}$ : standard region 1 . (b) $R_{12}$ : standard region 2 . (c) $R_{13}$ : standard region 3 with the parallel branch to be considered.

GridPiAnalyzer is further extended with the capability of our RRA approach for performance improvement. A new component is developed to decompose grid workflows into standard regions with parallel branches based on the procedure described in Fig.3. The formal verification is then recursively performed on each standard region instead of the whole grid workflow.

\subsection{Case Studies}

Let take our scenarios of gravitational wave data analysis as case studies. Along with the relatively simple workflow $S F 1$ introduced in Fig.2, two more complex ones $S F 2$ and $S F 3$ are also given in Figs. 5 and 6 . We only focus on the temporal properties verifications on the grid scientific workflows, thus the detail of its functionalities are not introduced here, which could be referred to [3].

Corresponding relaxed standard regions are decomposed in Figs. 7 and 8, respectively.

- $p 1$ : the necessary successor operations after template bank generation;

- $p 2$ : the constraints on working status of laser interferometers;

- $p 3$ : the completeness of incidental analysis;

- $p 4$ : the precondition of final incidental analysis.

Correspondingly the LTL-X formulae of these properties for each grid workflow are formulated in Table 1. These properties are required to be verified for all these three case studies.

\subsection{Performance Evaluation}

In this subsection, the proposed RRA approach is applied in formal verification of grid workflows and its performance is compared with several well-known verification methods ${ }^{[22]}$. These include the Symbolic Model Checking Algorithm (SMCA), SMCA with Cone of Influence (COI), SMCA with dynamic re-ordering of BDD (Binary Decision Diagram) variables (Dynamic), and Bounded Model Checking algorithm $(B M C(k)$, where $k$ is the setting of its length). All experiments are carried out using a machine with a Pentium IV $1.73 \mathrm{GHz}$ mobile CPU, 2.0 GB RAM, the Windows operating system and Eclipse development platform.

Performance evaluation results are illustrated in Figs. 9 and 10 in terms of verification time and peak memory usage, respectively.

The upper limit of verification time is $750 \mathrm{~s}$ for a complete grid workflow and $600 \mathrm{~s}$ for standard regions. The minimum considered peak memory usage is $10 \mathrm{Mb}$ during grid workflow verification. Among legends in Figs. 9 and 10, $S M C A^{\prime}$ indicates the time/memory usage for the model initialization with the $S M C A$ method. $S F 1, S F 2$, and $S F 3$ represent the three case studies illustrated in Figs. 2, 5 and 6, respectively. $R_{i j}$ indicates the verification on the $i$-th identified standard region in $S F_{j} . R R A$ indicates the verification result using the proposed approach. For a specific property $p$, the total verification time of $R R A\left(t_{p}\right)$ is computed as follows: $t_{p}=\sum_{i=1}^{n} t_{p}\left(R_{i}\right)$ where $t_{p}\left(R_{i}\right)$ is the verification 


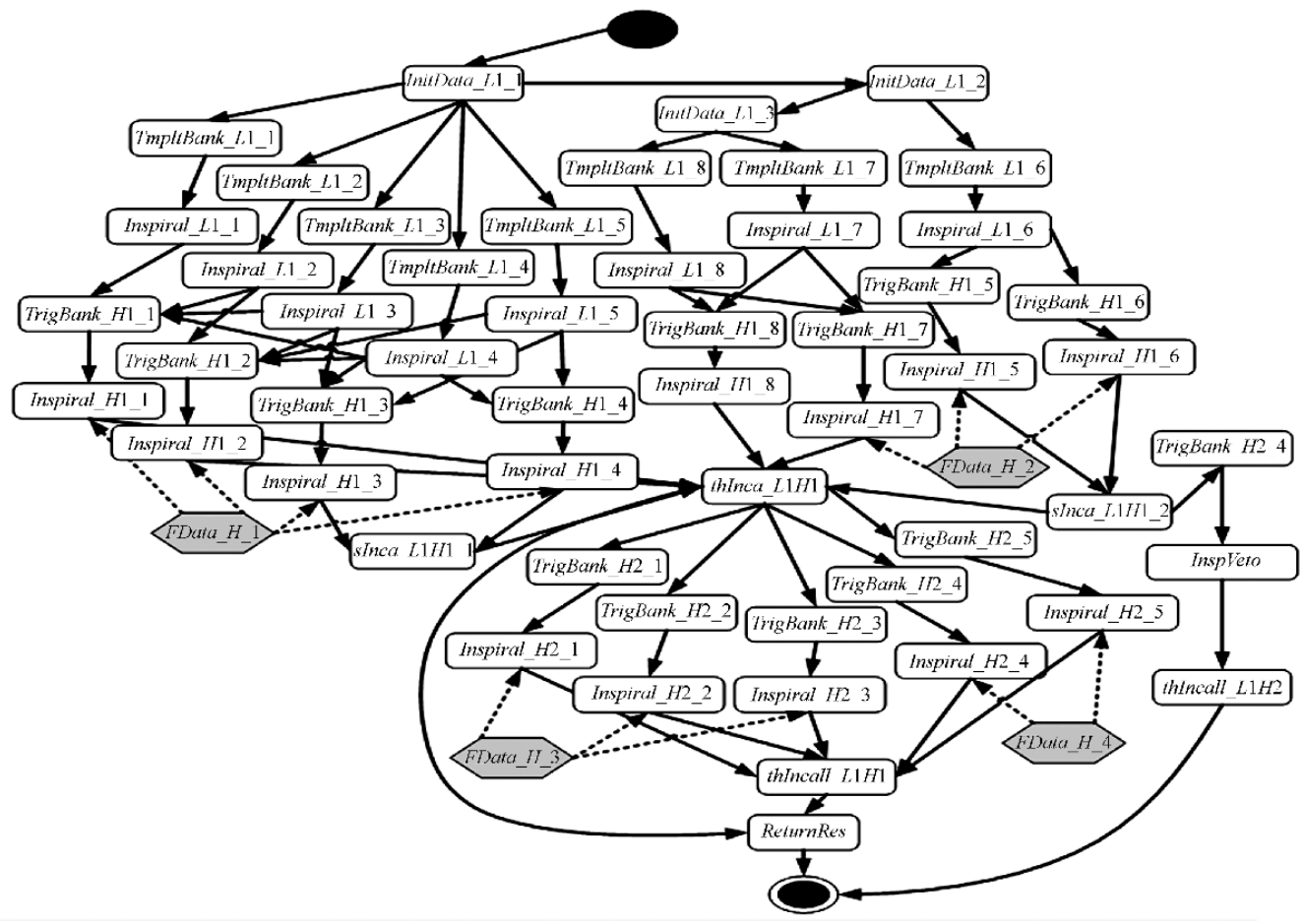

Fig.5. Gravitational wave data analysis - case study 2 (SF2).

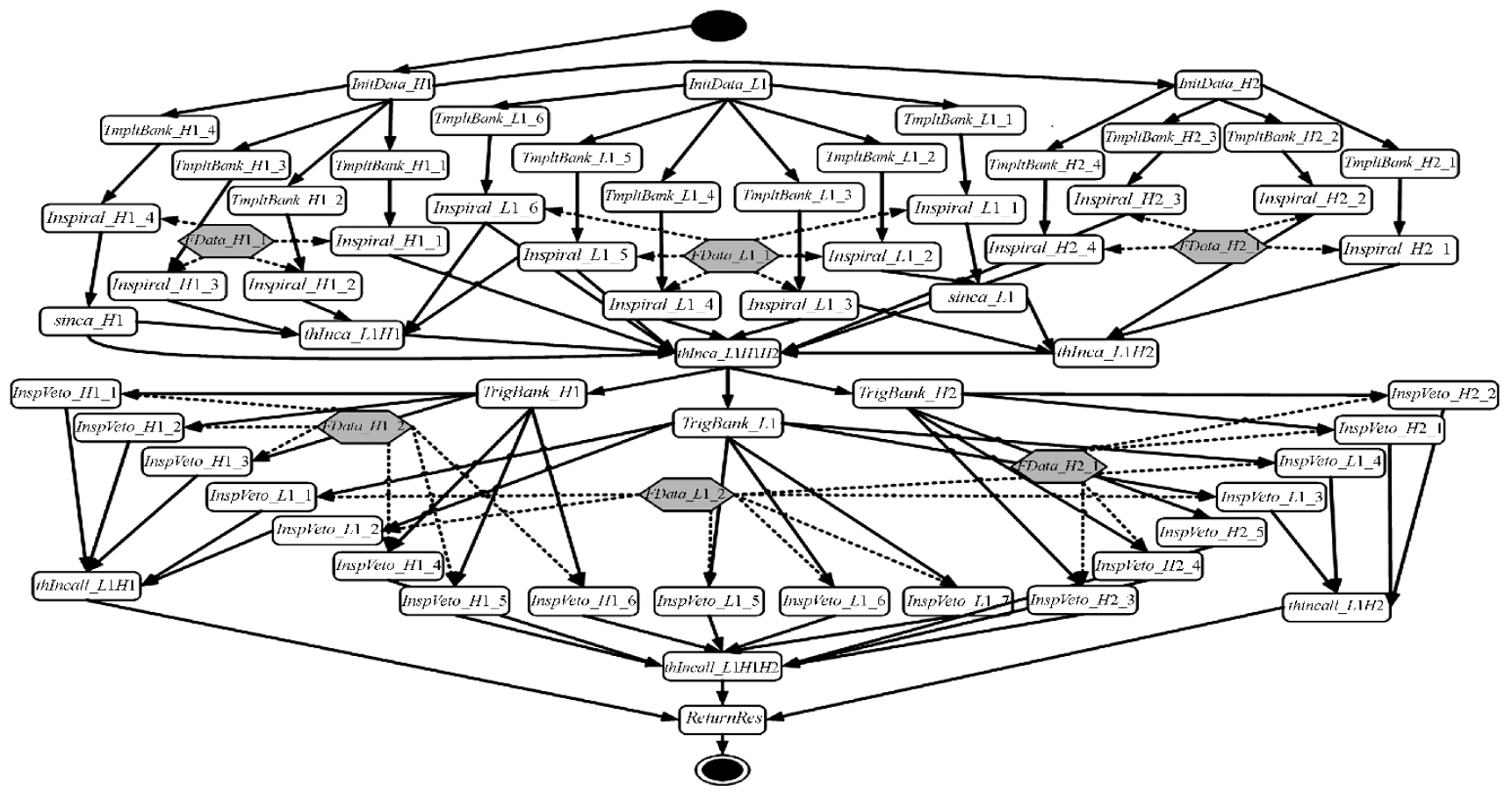

Fig.6. Gravitational wave data analysis - case study 3 (SF3).

time for $p$ on the $i$-th region in a grid workflow; the peak memory usage of $R R A\left(m_{p}\right)$ is computed as: $m_{p}=$ $\operatorname{Max}\left(m_{p}\left(R_{i}\right)\right)$ where $m_{p}\left(R_{i}\right)$ is the peak memory usage for $p$ on the $i$-th region in a grid workflow. The purpose of the additional Figs. 9(a) and 9(b) is to further zoom in the performance comparison with RRA and pure $S M C A / C O I$ approach in Fig.9(c).

From the results included in Figs. 9 and 10, it can be found that due to the complexity of the grid workflow $S F 2$ and SF3 (which contains $2^{\wedge} 14.5$ and $2^{\wedge} 15.4$ 


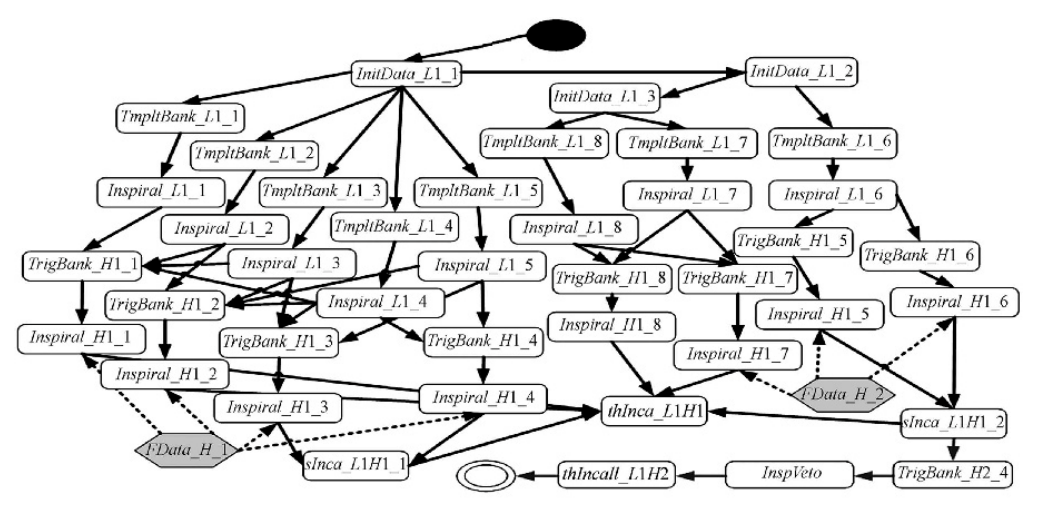

(a)
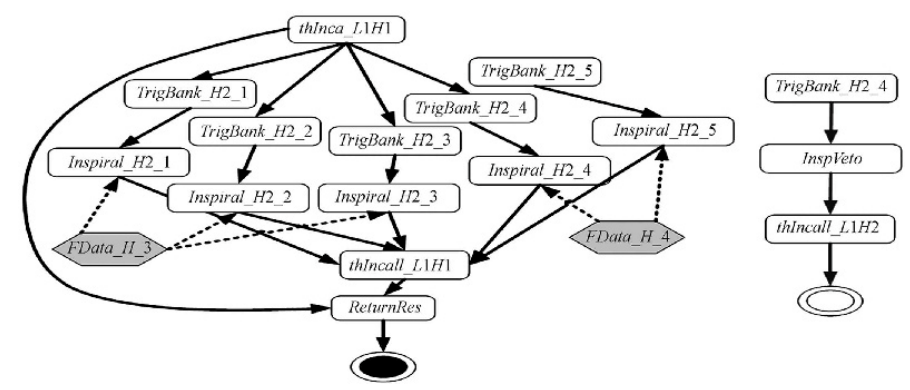

(b)

Fig.7. Relaxed region decomposition for $S F 2$. (a) $R_{21}$ : standard region 1. (b) $R_{22}$ : standard region 2 with the considered parallel branch.

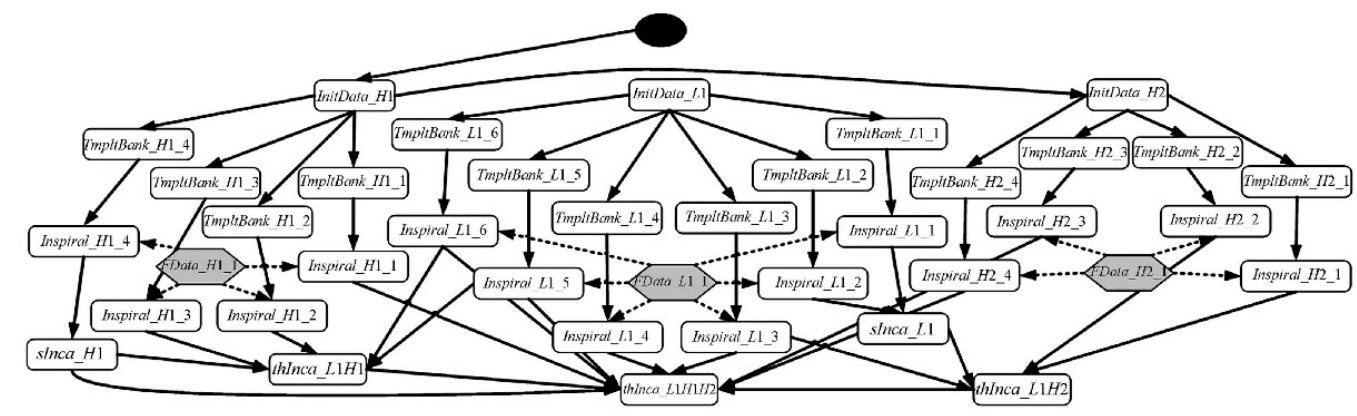

(a)

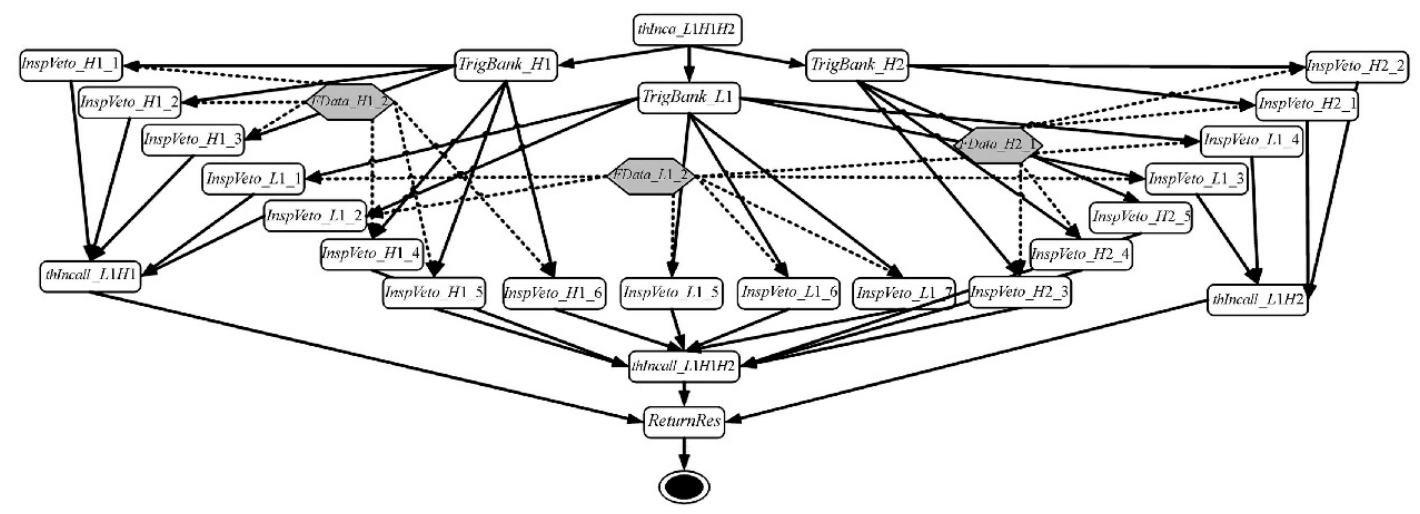

(b)

Fig.8. Relaxed region decomposition for SF3. (a) $R_{31}$ : standard region 1. (b) $R_{32}$ : standard region 2 . 
Table 1. Formulae of Properties Against $S F 1, S F 2$ and $S F 3$

\begin{tabular}{|c|c|c|}
\hline Prop. Name & Rarget SF & Formulae for the Desired Property \\
\hline$p 1_{1}$ & $S F 1 / S F 3$ & $\mathrm{G}($ TmpltBank_H1 $\rightarrow((\mathrm{F}$ TrigBank_H1) $\&(\mathrm{~F}$ Inspiral_H1 $)))$ \\
\hline$p 1_{2}$ & $S F 1 / S F 3$ & $\mathrm{G}($ TmpltBank_H2 $\rightarrow((\mathrm{F}$ TrigBank_H2)\&(F Inspiral_H2 $)))$ \\
\hline$p 1$ & $S F 2$ & $\mathrm{G}($ TmpltBank_L1 $\rightarrow((\mathrm{F}$ TrigBank_H1) $) \&(\mathrm{~F}$ Inspiral_H 1$)))$ \\
\hline \multirow[t]{2}{*}{$p 2$} & $S F 1 / S F 2$ & $\mathrm{G}(($ InitData_H $1 H 2) \rightarrow(\neg$ Inspiral_H2 U thInca_L $1 H 1))$ \\
\hline & SF3 & $\mathrm{G}(($ InitData_H1H2) $\rightarrow(\neg$ Inspiral_H2 U thInca_L1H1H2)$)$ \\
\hline \multirow[t]{2}{*}{$p 3$} & $S F 1 / S F 2$ & $\begin{array}{l}((\mathrm{F} \text { sInca_L } 1 H 1 \wedge(\neg \text { thIncall_L1H1U } \text { sInca_L } 1 H 1)) \vee \\
(\mathrm{F} \text { thInca_L } 1 H 1 \wedge(\neg \text { thIncall_L1H1 U thInca_L1H1)) }) \wedge \mathrm{F} \text { thIncall_L } 1 H 1\end{array}$ \\
\hline & $S F 3$ & 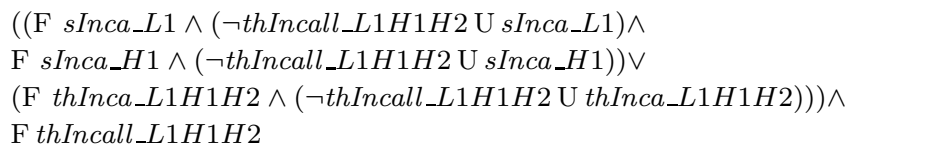 \\
\hline \multirow[t]{2}{*}{$p 4_{1}$} & $S F 1 / S F 2$ & $\mathrm{G}($ Inspiral_H1 $\rightarrow(\mathrm{F}$ thIncall_LL1H1) $)$ \\
\hline & $S F 3$ & $\mathrm{G}\left(\right.$ Inspiral_H$_{-} \mathrm{C} \rightarrow(\mathrm{F}$ thIncall_L $\left.L 1 H 1 H 2)\right)$ \\
\hline \multirow[t]{2}{*}{$p 4_{2}$} & $S F 1 / S F 2$ & $\mathrm{G}($ Inspiral_H $2 \rightarrow(\mathrm{F}$ thIncall_L $1 H 1))$ \\
\hline & SF3 & $\mathrm{G}\left(\right.$ Inspiral_$_{-} H 2 \rightarrow(\mathrm{F}$ thIncall_LL1H1H2) $)$ \\
\hline \multirow[t]{3}{*}{$p 4_{3}$} & $S F 1$ & $\mathrm{G}($ TmpltBank_H $1 \rightarrow(\mathrm{F}$ thIncall_LL1H1) $)$ \\
\hline & $S F 2$ & $\mathrm{G}($ TmpltBank_L1 $\rightarrow($ F thIncall_L$L 1 H 1))$ \\
\hline & SF3 & $\mathrm{G}($ TmpltBank_H1 $\rightarrow(\mathrm{F}$ thIncall_L1H1H2) $)$ \\
\hline \multirow[t]{2}{*}{$p 4_{4}$} & $S F 1$ & $\mathrm{G}\left(\right.$ TmpltBank_H2 $\rightarrow\left(\mathrm{F}\right.$ thIncall $\left.\left.\_L 1 H 1\right)\right)$ \\
\hline & SF3 & $\mathrm{G}($ TmpltBank_H2 $\rightarrow(\mathrm{F}$ thIncall_L $1 H 1 H 2))$ \\
\hline
\end{tabular}

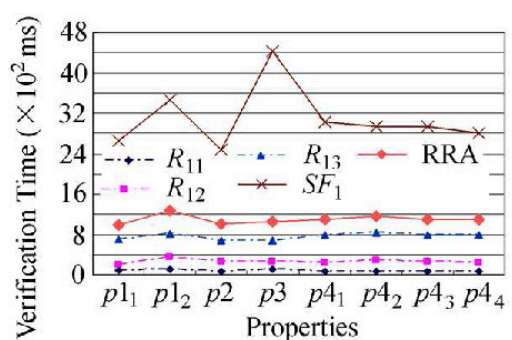

(a)

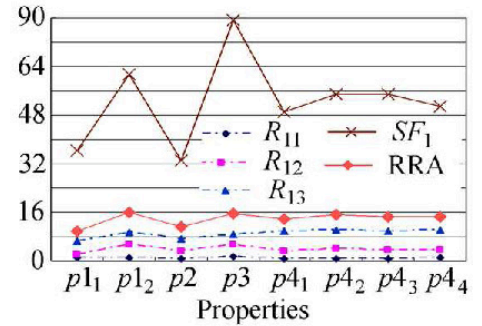

(b)

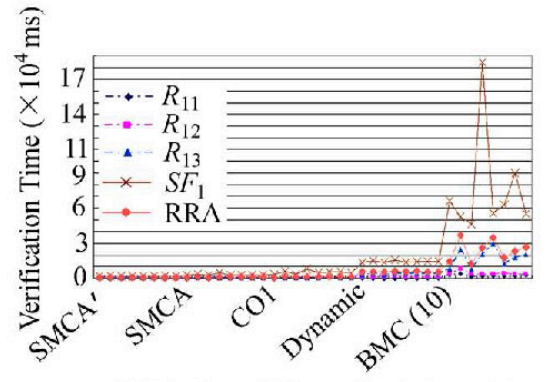

Methods and Properties $\left(p \mathrm{l}_{1} \sim p 4_{4}\right)$

(c)

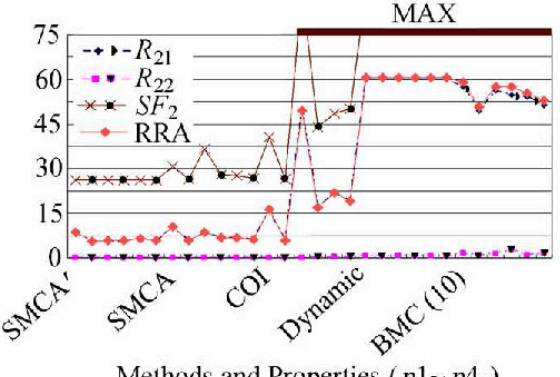

Methods and Properties $\left(p 1 \sim p 4_{3}\right)$

(d)

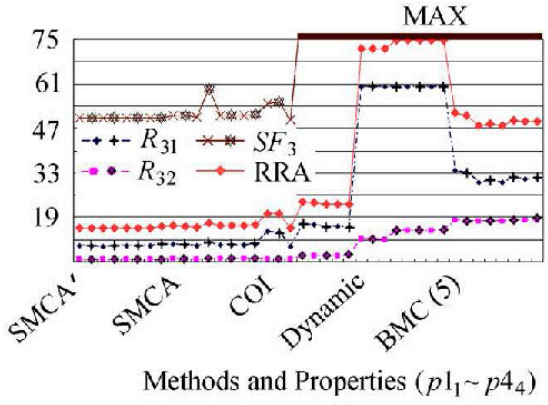

(e)

Fig.9. Performance evaluation of verification time for $S F 1, S F 2$ and $S F 3$. (a) SMCA method on $S F 1$. (b) SMCA + CoI methods on $S F 1$. (c) All methods on $S F 1$. (d) All methods on $S F 2$. (e) All methods on SF3.

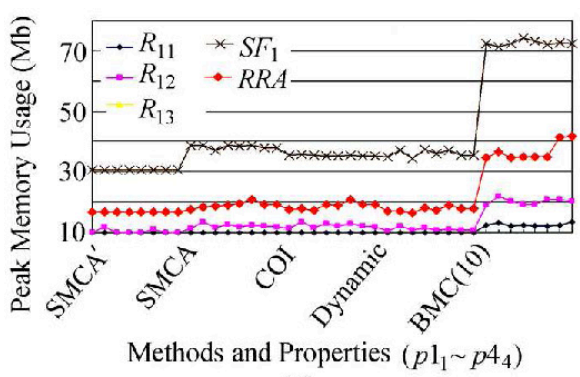

(a)

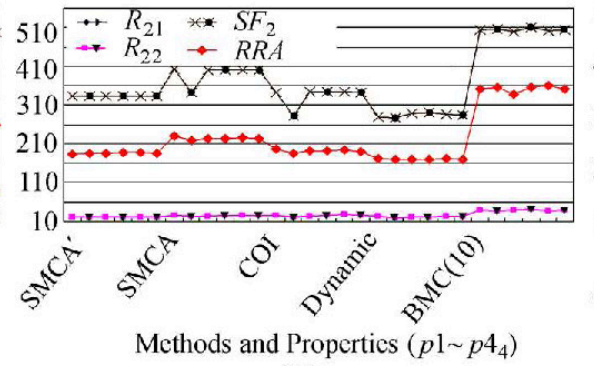

(b)

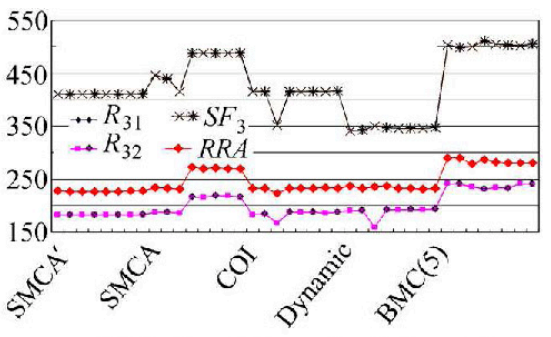

Methods and Properties $\left(p 1_{1} \sim p 4_{4}\right)$

(c)

Fig.10. Performance evaluation of perk memory usage. (a) All methods on $S F 1$. (b) All methods on $S F 2$. (c) All methods on $S F 3$. 
reachable states, respectively), direct verification of these workflows with none of the compared methods shows satisfactory performance. Based on SMCA and $C O I$, verification time exceeds $250 \mathrm{~s}$ and $500 \mathrm{~s}$ respectively; peak memory usage exceeds $400 \mathrm{Mb}$ and $480 \mathrm{Mb}$ respectively. Based on Dynamic and $B M C$, verification time even exceeds the upper limit and peak memory assumption exceeds $290 \mathrm{Mb}, 510 \mathrm{Mb}, 349 \mathrm{Mb}$ and $511 \mathrm{Mb}$, respectively. Poor performance of the $B M C$ approach is partially due to the use of the simple Mini-SAT solver. Performance comparison between $B M C$ and $S M C A$ is out of the scope of this paper.

Based on our proposed RRA approach, the verification time is reduced to over $60 \mathrm{~s}, 160 \mathrm{~s}, 590 \mathrm{~s}$ and $480 \mathrm{~s}$ for $S F 2$ and $S F 3$ in comparison with the $S M C A, C O I$ and $B M C$ method respectively. The peak memory usage is dramatically reduced to $230 \mathrm{Mb}, 196 \mathrm{Mb}, 171 \mathrm{Mb}$, and $360 \mathrm{Mb}$ for $S F 2$ and $270 \mathrm{Mb}, 232 \mathrm{Mb}, 236 \mathrm{Mb}$, $290 \mathrm{Mb}$ for $S F 3$. Here for RRA with the Dynamic method, the verification time for the first standard region in $S F 2$ and $S F 3$ still exceeds the upper limit. This is because the Dynamic method is in essential a memory saving optimization technique which may worsen the verification efficiency.

By applying the RRA approach, memory usage savings are guaranteed since RRA enables partial loading and verification of grid workflows. Since state spaces of separate regions are also reduced compared to the global workflow, the proposed RRA approach not only reduces verification time, but also the time for BDD operation, Boolean satisfiability solving, memory operations, etc. in the real implementation of $S M C A$ and $B M C$ approaches. These result in global performance improvement for the proposed RRA approach.

\section{Conclusions}

In this work, We propose state $\mathrm{Pi}$ calculus, an extension of the existing Pi calculus for formal representation of grid services. Formalism of different activities and control structures using state Pi calculus is implemented to describe scientific workflows composed with grid services.

Furthermore, the decomposition strategy for standard regions-based verification of grid workflows is proposed to enhance the performance in the formal verification of grid workflow correctness. The RRA approach can effectively decompose a grid workflow into separate standard regions with parallel branches. Consequently, costly global reasoning of a grid workflow can be decomposed into light-weight local reasoning of its standard regions, each with a reduced state space.

The approach is implemented in our GridPiAnalyzer, an automatic formal verification system. Detailed experimental results show that the verification overhead in terms of both CPU time and memory usage are dramatically reduced by applying our RRA approach, compared with using various traditional formal verification algorithms directly.

The disadvantages of this work is that it works on single workflow only, without considering more on multiple grid workflows that interact with each other. However this problem can be overcomed by a formal representation of multiple interactive workflows using state Pi calculus, with introducing more semantics regulations.

Our ongoing work includes refinement of the GridPiAnalyzer system, with the implementation of more grid workflow verification modules, especially interactive multiple workflow systems. Applying the RRA approach to more and more complex real world applications is also our future research direction.

\section{References}

[1] Foster I, Kesselman C. The Grid: Blueprint for a New Computing Infrastructure. San Fransisco: Morgan-Kaufmann, 1998.

[2] Cao J, Jarvis S A, Saini S, Nudd G R. GridFlow: Workflow management for grid computing. In Proc. the 3rd IEEE/ACM Int. Symp. on Cluster Computing and the Grid, Tokyo, Japan, May 12-15, 2003, pp.198-205.

[3] Brown D A, Brady P R et al. A case study on the use of workflow technologies for scientific analysis: Gravitational wave data analysis. In Workflows for eScience: Scientific Workflows for Grids, Taylor I J, Dealman E, Gannon D B et al (eds.), Springer Verlag, 2007, pp.39-59.

[4] Cao J, Fingberg J, Berti G et al. Implementation of gridenabled medical simulation applications using workflow techniques. In Proc. the 2nd Int. Workshop on Grid and Cooperative Computing, Shanghai, China, Dec. 7-10, 2003, pp.34-41.

[5] Liu Y, Müller S, Xu K. A static compliance checking framework for business process models. IBM Systems Journal, 2007, 46(2): 335-362.

[6] Chen J, Yang Y. A taxonomy of grid workflow verification and validation. Concurrency and Computation: Practice and Experience, 2008, 20(4): 347-360.

[7] Chen J, Yang Y. Multiple states based temporal consistency for dynamic verification of fixed-time constraints in grid workflow systems. Concurrency and Computation: Practice and Experience, 2007, 19(7): 965-982.

[8] Tan W, Fan Y, Zhou M. A petri net-based method for compatibility analysis and composition of Web services in business process execution Language. IEEE Transactions on Automation Science and Engineering, 2009, 6(1): 94-106.

[9] Tan W, Fan Y, Zhou M et al. Data-driven service composition in enterprise SOA solutions: A petri net approach. IEEE Trans. Automation Science and Engineering, 2010, 7(3): 686-694.

[10] Li X, Fan Y, Sheng Q Z et al. A petri net approach to analyzing behavioral compatibility and similarity of Web services. IEEE Trans. Systems, Man, and Cybernetics, Part A: Systems and Humans, 2010, 41(3): 510-521.

[11] Xiong P, Fan Y, Zhou M. Web service configuration under multiple quality-of-service attribute. IEEE Trans. Automation Science and Engineering, 2009, 6(2): 311-321. 
[12] Xiong P, Fan Y, Zhou M. QoS-aware Web service configuration. IEEE Trans. Systems, Man and Cybernetics, Part A, 2008, 38(4): 888-895.

[13] Clarke E M, Grumberg O, Peled D A. Model Checking, MIT Press, 1999.

[14] Xu K, Wang Y X, Wu C. Formal verification technique for grid service chain model and its application. Science in China, Series F: Information Sciences, 2007, 50(1): 1-20.

[15] Xu K, Cao J, Liu L, Wu C. Performance optimization of temporal reasoning for grid workflows using relaxed region analysis. In Proc. the 22nd IEEE Int. Conf. Advanced Information Networking and Applications Workshops, GinoWan, Japan, March 25-28, 2008, pp.187-194.

[16] Salaün G, Bordeaux L, Schaerf M. Describing and reasoning on Web services using process algebra. In Proc. Int. Conf. Web Services, San Diego, USA, June 6-9, 2004, pp.43-50.

[17] Németh Z, Sunderam V. Characterizing grids: Attributes, definitions, and formalisms. J. Grid Computing, 2003, 1(1): 9-23.

[18] Huang S, Mulcahy J J. Software reuse in the evolution of an e-commerce system: A case study. International Journal of Computing \& Information Technology, 2(1): 1-15.

[19] Cai H. Scale-free Web services. In Proc. Int. Conf. Web Services, Salt Lake City, USA, July 9-13, 2007, pp.288-295.

[20] Milner R. Communicating and Mobile Systems: the Pi Calculus. Cambridge University Press, 1999.

[21] Wang S, Armstrong M P. A quadtree approach to domain decomposition for spatial interpolation in grid computing environments. Parallel Computing, 2003, 29(10): 1481-1504.

[22] Cimatti A, Clarke E et al. NuSMV2: An open source tool for symbolic model checking. In Proc. the 14th Int. Conf. Computer Aided Verification, Copenhagen, Denmark, July 27-31, 2002, 359-364.

[23] Deelman E, Kesselman C et al. GriPhyN and LIGO, building a virtual data grid for gravitational wave scientists. In Proc. the 11th Int. Symp. High Performance Distributed Computing, Edinburgh, Scotland, July 24-26, 2002, pp.225-234.

[24] Liu R, Kumar A. An analysis and taxonomy of unstructured workflows. In Proc. the 3rd Int. Conf. Business Process Management, Nancy, France, Sept. 5-9, 2005, pp.268-284.

[25] Grumberg O, Long D E. Model checking and modular verification. ACM Transactions on Programming Languages and Systems, 1999, 16(3): 843-871.

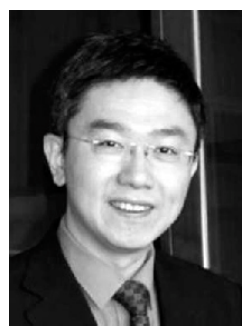

Jun-Wei Cao received his Ph.D. degree in computer science from the University of Warwick, Coventry, UK, in 2001. He received his B.S. and M.S. degrees in control theories and engineering in 1996 and 1998, respectively, both from Tsinghua University, Beijing, China. He is currently a professor and vice director, Research Institute of Information Technology, Tsinghua University. He is also with Tsinghua National Laboratory for Information Science and Technology. He is now a visiting scientist of MIT. Before joining Tsinghua University in 2006, he was a research scientist at MIT LIGO Laboratory and NEC Laboratories Europe for about 5 years. He has published over 120 papers, which have been cited by international scholars for over 2200 times. He has authored or edited 5 books and proceedings. His research is focused on advanced computing technologies and applications. Prof. Cao is a Senior Member of the IEEE Computer Society and a Member of the ACM and CCF.

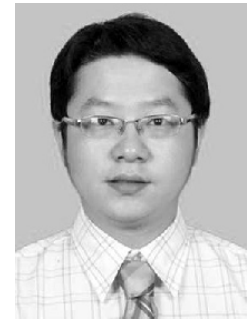

Fan Zhang received the B.S. degree in computer science from Hubei Univ. Technology and M.S. degree in control science and engineering from Huazhong University of Science and Technology. He is currently a Ph.D. student in the Department of $\mathrm{Au}$ tomation, Tsinghua University. His research interests include data center networks and grid/cloud computing.

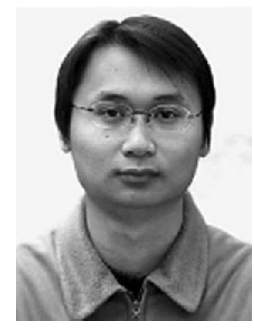

$\mathrm{Ke} \mathbf{X u}$ is a research engineerer in Morgan Stanley, Shanghai, China. $\mathrm{He}$ received his B.S. degree in 2002 from Automation Department of Shanghai Jiao Tong University, China and Ph.D. degree from the National CIMS Engineering Research Center in Tsinghua University in 2007. His main research interests include grid computing, business integration, formal methods, and model checking. He is also an IBM Ph.D. fellow in year 2006-2007.

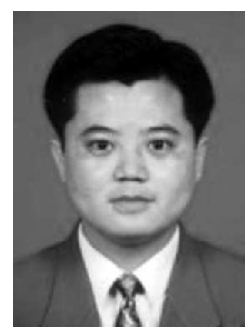

Lian-Chen Liu is currently an associate professor of the Department of Automation, Tsinghua Univ. $\mathrm{He}$ received the Ph.D. degree from Nankai Univ., China. His research interests include large scale scientific resource sharing, distributed computing.

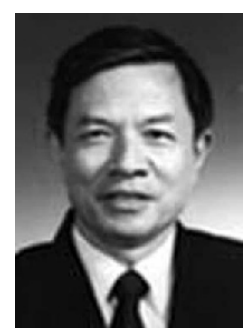

Cheng $\mathbf{W u}$ is a professor of the Department of Automation, Tsinghua Univ., director of National CIMS Engineering Research Center, and member of the Chinese Academy of Engineering. He received his B.S. and M.S. degrees from the Department of Automation, Tsinghua Univ. in 1962 and 1966 respectively. His research interests include complex manufacturing system scheduling, grid/cloud applications. 